\title{
Post-traumatic headache: facts and doubts
}

\author{
Rita Formisano • Umberto Bivona • \\ Sheila Catani · Mariagrazia D'Ippolito • \\ M. Gabriella Buzzi
}

Received: 15 December 2008/ Accepted: 3 February 2009/Published online: 18 March 2009

(C) Springer-Verlag 2009

\begin{abstract}
The International Classification of Headache Disorders does not separate the moderate from severe/very severe traumatic brain injury (TBI), since they are all defined by Glasgow coma scale (GCS) $<13$. The distinction between the severe and very severe TBI $($ GCS $<8$ ) should be made upon coma duration that in the latter may be longer than 15 days up to months in the case of vegetative state. Post-traumatic amnesia duration may double the coma duration itself. Therefore, the 3-month parameter proposed to define the occurrence or resolution of posttraumatic headache (PTH) appears inadequate. Following TBI, neuropathic pain, central pain, thalamic pain, combined pain are all possible and they call for proper pharmacological approaches. One more reason for having difficulties in obtaining information about headache in the early phase after regaining consciousness is the presence of concomitant medications that may affect pain perception. Post-traumatic stress disorder (PTSD) develops days or weeks after stress and tends to improve or disappear within 3 months after exposure; interestingly, this spontaneous timing resembles that of PTH. In our experience the number of TBI patients with PTH at 1-year follow-up is lower in those with longer coma duration and more severe TBI. Cognitive functioning evaluated after at least 12 months from TBI, showed mild or no impairment in these patients with severe TBI and PTH, whereas they have psychopathological changes, namely anxiety and depression. The majority of patients with PTH after severe/very
\end{abstract}

R. Formisano $(\bowtie) \cdot$ U. Bivona · S. Catani · M. D’Ippolito · M. G. Buzzi

Post-Coma Unit and Headache Center, IRCCS Fondazione Santa Lucia, Via Ardeatina 306, 00179 Rome, Italy

e-mail: r.formisano@hsantalucia.it severe TBI had skull fractures or dural lacerations and paroxystic EEG abnormalities. The combination of psychological changes (depression and anxiety) and organic features (skull fractures, dural lacerations, epileptic EEG abnormalities) in PTH may be inversely correlated with the severity of TBI, with prevalence of psychological disturbances in mild TBI and of organic lesions in severe TBI. On the other hand, only in severe TBI patients with good cognitive recovery the influence of the psychopathological disorders may play a role. In fact, the affective pain perception is probably related to the integrity of cognitive functions as in mild TBI and in severe TBI with good cognitive outcome.

Keywords Headache classification .

Post-traumatic headache - Severe traumatic brain injury . Cognitive functioning

\section{Introduction}

The International Classification of Headache Disorders (ICHD-II) [1] is intended to provide clinical criteria to classify primary and secondary headaches and to serve for adequate and homogeneous diagnoses among experts. It provides up to four sophistication levels of diagnosis and therefore it appears that any pain located in the head and neck may be posed into the proper box. Even in the lack of discrete criteria, clinical entities can be classified, given that the probability is satisfied.

Although the classification has given a great opportunity of speaking a common language, it no longer seems satisfactory. In fact, one of the major pitfalls of the classification is represented by the lack of further clinical and pharmacological data, which are becoming 
more and more common in clinical practice due to the thorough evaluation of patients. For example, the debate concerning chronic headaches [2] calls for exhaustive examination of the patients in the aim of disclosing psychological features that may provide other characteristics and criteria to define psychological disturbances underlying drug abuse and possibly identify comorbid conditions that contribute to chronic pain syndromes [3]. Another example is represented by the presence of premonitory symptoms in migraine attacks that is large enough to identify migraine subpopulation and possibly tailored treatment, according to the underlying neurotransmission mechanisms involved in premonitory symptoms pathophysiology $[4,5]$.

Classification and description of post-traumatic headache (PTH), as classified in the ICHD-II are even more scant. The issue will open up an important debate, if only more attention is paid to the many clinical features that characterize the post-traumatic syndrome. PTH is, in fact, classified according to the sole temporal and, partly, neuroimaging data regarding head trauma, without any description of pain characteristics. Most of the issues regarding PTH remain controversial [6] and need more epidemiological, clinical and pathophysiological studies.

This short review will focus on the paucity of information about headache following head trauma and will try to outline the needs for accurate research in this field to provide future revisions of the present guidelines.

\section{Post-traumatic headache: the facts and the doubts}

\section{The ICHD-II criteria}

Criteria for diagnosing headaches following head trauma, according to the ICHD-II are shown in Table 1 [1]. According to them, PTH may have an acute or chronic pattern and either acute or chronic forms may occur after severe to moderate or mild traumatic brain injury (TBI).

According to Baandrup and Jensen [7] the ICHD-II did not add further to the previous IHS guidelines [8], therefore suggesting that characterization of PTH and research into this area is scarce. The authors investigated PTH in 53 patients who sustained mild $(n=45)$ or moderate/severe $(n=8)$ TBI and evaluated the presence of medication overuse. Data are shown in Table 2.

Following authors' comment, the cohort of patients reported did fulfil the diagnostic criteria both for ICHD-II and the previous edition, indicating that the new restriction provided in ICHD-II have non-significant influence on the great majority of patients with chronic PTH.

\section{Post-traumatic headache after moderate/severe and very severe head injury}

Post-traumatic amnesia (PTA) is generally defined as the time interval from head trauma to the recovery of memory of the current events and of those occurring in the last $24 \mathrm{~h}$ [9]. PTA may last from two to four times the coma duration itself [10]. Therefore, the ICDH-II criterion of the 7 day interval after regaining consciousness for PTH development may be inadequate, since the mental confusion and impaired communication during PTA limit the subjects ability to complain or to be aware of headache until PTA resolution.

The ICHD-II does not separate the severe from the very severe TBI, since both are defined by Glasgow coma scale (GCS) $<8$ [11], whereas the distinction should be made upon coma duration $[12,13]$. This observation is more than academic. In fact, the very severe TBI may lead to a coma duration of more than 15 days up to months in the case of vegetative state and the PTA duration may double the coma duration itself [14]. Therefore, the 3-month parameter proposed to define the occurrence or resolution of PTH appears inadequate, especially in those most severe TBI cases with prolonged disturbance of consciousness and PTA lasting several months.

For as moderate/severe TBI is concerned, the ICHD-II criteria suggest that imaging data refer to the acute phase, but do not provide any clue for the follow-up period. The latest issue rises further consideration regarding the occurrence of delayed pain after TBI and the quality of such pain. In fact, pain pathways can be damaged at any location following head trauma, from the very peripheral structures (bone, muscles and nerves) to the highest cortical, subcortical and brainstem level. Therefore, any pain quality is possible and that should be carefully evaluated and treated accordingly: neuropathic pain, central pain, thalamic pain, combined pain are all possible and they call for proper pharmacological approaches. Moreover, imaging data in the chronic phase after TBI may be useful in order to evaluate the progressive degeneration of pathways, or their functional recovery, to understand the fate of many deficits and symptoms, pain being one of them, in the long run.

According to the ICHD-II, acute PTH occurs after 1 week from head trauma and may cease within 3 months (acute headache) or later (chronic). This definition is evidently discrepant with the clinical feature of very severe TBI patients, since loss of consciousness may persist for several weeks (or months) and PTA is rather longer. Unfortunately, the ICHD-II criteria do not allow separate diagnosing between moderate/severe and very severe TBI. Therefore, the temporal pattern provided in the guidelines appears inadequate and not applicable to all patients, as said above. 
Table 1 The International Classification of Headache Disorders (ICHD-II): post-traumatic headache

\subsection{Acute post-traumatic headache}

\subsubsection{Acute post-traumatic headache attributed to moderate or severe head injury}

Diagnostic criteria

A. Headache, no typical characteristics known, fulfilling criteria C and D

B. Head trauma with at least one of the following

1. loss of consciousness for $>30 \mathrm{~min}$

2. Glasgow coma scale $(\mathrm{GCS})<13$

3. post-traumatic amnesia for $>48 \mathrm{~h}$

4. imaging demonstration of a traumatic brain lesion (cerebral haematoma, intracerebral and/or subarachnoid haemorrhage, brain contusion and/or skull fracture)

C. Headache develops within 7 days after head trauma or after regaining consciousness following head trauma

D. One or other of the following:

1. headache resolves within 3 months after head trauma

2. headache persists but 3 months have not yet passed since head trauma

5.1.2 Acute post-traumatic headache attributed to mild head injury

Diagnostic criteria

A. Headache, no typical characteristics known, fulfilling criteria $\mathrm{C}$ and $\mathrm{D}$

Head trauma with all the following

1. either no loss of consciousness, or loss of consciousness of $<30$ min duration

2. Glasgow coma scale $(\mathrm{GCS}) \geq 13$

3. symptoms and/or signs diagnostic of concussion

Headache develops within 7 days after head trauma

D: One or other of the following

1. headache resolves within 3 months after head trauma

2. headache persists but 3 months have not yet passed since head trauma

5.2 Chronic post-traumatic headache

5.2.1 Chronic post-traumatic headache attributed to moderate or severe head injury

Diagnostic criteria

A. Headache, no typical characteristics known, fulfilling criteria $\mathrm{C}$ and $\mathrm{D}$

B. Head trauma with at least one of the following

1. loss of consciousness for $>30 \mathrm{~min}$

2. Glasgow coma scale $(\mathrm{GCS})<13$

3. post-traumatic amnesia for $>48 \mathrm{~h}$

4. imaging demonstration of a traumatic brain lesion (cerebral haematoma, intracerebral and/or subarachnoid haemorrhage, brain contusion and/or skull fracture)

C. Headache develops within 7 days after head trauma or after regaining consciousness following head trauma

D. Headache persists for $>3$ months after head trauma

5.2.2 Chronic post-traumatic headache attributed to mild head injury

Diagnostic criteria

A. Headache, no typical characteristics known, fulfilling criteria C and D

B. Head trauma with all the following

1. either no loss of consciousness, or loss of consciousness of $<30$ min duration

2. Glasgow coma scale (GCS) $\geq 13$

3. symptoms and/or signs diagnostic of concussion

C. Headache develops within 7 days after head trauma

D. Headache persists for $>3$ months after head trauma
One more reason for having difficulties in obtaining information about headache in the early phase after regaining consciousness, especially when TBI was severe or very severe, is the presence of concomitant medications. In fact, these patients receive antiepileptic therapy to treat behavioural disturbances and post-traumatic seizures [15] 
Table 2 Evaluation of posttraumatic headache clinical features and medication overuse in TBI patients, according to ICHD-II [7]

\begin{tabular}{lcc}
\hline Headache clinical features & No. of patients & $\begin{array}{c}\text { Medication overuse } \\
\text { (No. of patients) }\end{array}$ \\
\hline Episodic tension-type headache & 6 & 0 \\
Chronic tension-type headache & 27 & 12 \\
Migraine without aura & 1 & 1 \\
Chronic tension-type headache + Migraine without aura & 4 & 3 \\
Chronic tension-type headache + Probable migraine & 10 & 5 \\
Non-classified & 5 & 1 \\
Total & 53 & 22 \\
\hline
\end{tabular}

or beta-blockers, namely propranolol, which is the only effective drug in psychomotor agitation, according to Cochrane Analysis [16], which is also used for post-traumatic dysautonomic syndrome, all drugs that may affect pain perception. Anticonvulsants or GABAergic drugs such as gabapentin, pregabalin or clonazepam, which are given to reduce central or peripheral pain or indomethacin to prevent para-articular ossification [17], may well be responsible for attenuation of head pain, if present.

Severe TBI does not only imply cerebral damage. In fact, the trauma may be responsible for skull fractures and epidural, subdural and intracerebral haemorrhage may occur. The latest conditions often require neurosurgical procedures and therefore certain causes of head pain should be classified along with the pure PTH, as shown in bold in Table 3.

Skull fractures are often associated with dural laceration and tearing. Surgical craniotomy to evacuate haematoma or to reduce intracranial blood pressure in the acute phase of TBI produce dural interruption and scar formation. Lesion of the dura mater may produce neurogenic inflammation due to depolarization of sensory fibres and contribute to the antidromic activation of nociceptive pathways mediated by the trigemonovascular system [18]. Haemoglobin from haemorrhage (either intracerebral or epi-subdural haematoma) may be responsible for dural sensitive innervation activation [19]. A chronic damage of dura and attendant sensory fibres may contribute to sensitisation and pain production [20, 21].

A late complication of TBI is represented by hydrocephalus which is, itself, a possible cause of headache. Beside of the presence of headache, hydrocephalus should always be sought after TBI in patients in whom recovery of either motor or cognitive functioning course during rehabilitation declines or there is worsening of functions [22].

A recent longitudinal study among 109 moderate/severe TBI patients [23], showed that in the acute rehabilitation phase, $37.6 \%$ of patients developed PTH. Among them, PTH at 6 months from regaining consciousness was still present in $20.2 \%$ patients and in $19.3 \%$ of patients at 12 months. Despite of the careful description of PTH occurrence at different time points, and the quantitative
Table 3 Other causes of headache following head injury, according to ICHD-II

\section{Headache attributed to head and/or neck trauma \\ 5.1 Acute post-traumatic headache \\ 5.1.1 Acute post-traumatic headache attributed to moderate or severe head injury \\ 5.1.2 Acute post-traumatic headache attributed to mild head injury \\ 5.2 Chronic post-traumatic headache \\ 5.2.1 Chronic post-traumatic headache attributed to moderate or severe head injury \\ 5.2.2 Chronic post-traumatic headache attributed to mild head injury \\ 5.3 Acute headache attributed to whiplash injury \\ 5.4 Chronic headache attributed to whiplash injury}

5.5 Headache attributed to traumatic intracranial haematoma

5.5.1 Headache attributed to epidural haematoma

5.5.2 Headache attributed to subdural haematoma

5.6 Headache attributed to other head and/or neck trauma

5.6.1 Acute headache attributed to other head and/or neck trauma

5.6.2 Chronic headache attributed to other head and/or neck trauma

5.7 Post-craniotomy headache

5.7.1 Acute post-craniotomy headache

5.7.2 Chronic post-craniotomy headache 
method to measure PTH in this cohort, authors failed to obtain premorbid data to analyse along with recovery, in order to verify the hypothesis that a pre-existent headache may have been hidden by poor cognitive functioning recovery. In fact, nociception per se may not be reduced, whereas the poor affective component of pain perception may contribute to the lack or low pain experience in those patients.

The fate of pre-traumatic headache after TBI is uneven, depending to cognitive recovery. In our experience some patients may refer a transient or definitive relief of pain after severe TBI, although generally a good cognitive recovery is followed by reappearance of pre-existing headache. Other issues should be also considered. For example, in women, recovery of hormonal fluctuations occurs months later (up to 1 year) following TBI and the recovery of such physiological fluctuations may contribute to brain susceptibility to experience migraine pain again. Besides, too many factors which are responsible for secondary pain due to TBI interfere with previous primary headache pattern. Efforts should, therefore, be made to identify pain syndromes in order to provide relief other than try to classify them within strict criteria.

Baandrup and Jensen [7] in their review report that they "could not find many of the so-called late-acquired posttraumatic headache, indicating that this subgroup is not as common as earlier assumed".

As personal comment, it may be that this subgroup consists of those very severe TBI, with prolonged disturbances of consciousness, longest PTA, late recovery of cognitive and affective functions and therefore later complaining of PTH.

Neuropsychological and cognitive functioning after moderate, severe and very severe TBI

In our experience, acute PTH after moderate, severe and very severe TBI is not a proper definition, since patients may complain of PTH months after severe TBI and this is true only for those subjects who regained an adequate cognitive functioning and therefore affective pain perception along with high-level integration, that serve to discriminate a discomfort as head pain with its given characteristics. It is unreal to expect that the havoc those patients go through allow such a sophisticated discrimination and in fact, the number of severe TBI patients who experience a clear head pain are the very minority.

Based on our clinical experience on PTH after severe and very severe TBI we retrospectively compared two different TBI patient groups and we found that the number of patients with headache at 1-year follow-up was lower in those with longer coma duration and more severe TBI (Table 4) (data published in abstract form, [24, 25].
Table 4 Comparison between severe and very severe TBI and headache at follow-up [24, 25]

Severe TBI: GCS $\leq 8$; coma duration 3-14 days $-n=77$

no. of patients with chronic PTH 18/77 (23.4\%)

Very severe TBI-GCS $\leq 8$ - Coma duration $>15-60$

days $-n=300$

no. of patients with chronic PTH 13/300 (4.3\%)

As shown in Table 4, the occurrence of PTH in very severe TBI was $4.3 \%$ (13 patients). All headaches in this group had features of primary-like headaches (migraine without aura, 31\%; chronic tension-type headache, 69\%). Only one patient had both. Cognitive functions, detected through neuropsychological evaluation after at least 12 months from TBI, showed mild or no impairment. In the same group of patients, psychopathological changes were found: anxiety-depression were present in $57 \%$ of the patients; depression alone was diagnosed in $29 \%$ and anxiety in $14 \%$ of patients. Beside of the association with good cognitive recovery, depression and anxiety, the majority of patients $(69.1 \%)$ with PTH had skull fractures or dural lacerations (traumatic or due to craniotomy) and paroxystic EEG abnormalities with specific sharp waves $(84.6 \%)$.

It is noteworthy that headache associated to anxiety and/ or depression may represent an adaptive disorder syndrome to TBI and its sequelae. Therefore, TBI of any severity should be studied as a possible trigger of post-traumatic stress disorder (PTSD). PTSD may develop in any person who experiences extreme stress with fear, helplessness or horror following threat of injury or death [26]. Therefore, TBI may well be responsible for the development of any of the three symptom clusters characterizing PTSD: re-experiencing symptoms, avoidance symptoms and hyper-arousal symptoms, all being a possible cause of stress-related headache. PTSD develops days or weeks after stress and tends to improve or disappear within 3 months after exposure to the stressful event or after PTA resolution in most severe cases [27]. Interestingly, this spontaneous timing resembles that of PTH. The co-occurrence of PTSD and TBI is intriguing [28]. Interestingly, mild TBI is less protective toward PTSD, compared to severe TBI. Again, PTSD and TBI sequelae (such as PTH), call for concomitant diagnosis and treatment. Some recent studies reported that patients who sustain a severe TBI and are fully amnesic of their trauma could nevertheless develop PTSD [29, 30].

\section{Post-traumatic headache after mild head injury}

Pain may occur after head trauma and in most of cases it is not the only symptom. In fact, it may be associated with 
dizziness, difficulty in concentration, nervousness, personality changes and insomnia, etc. Taken together, these symptoms characterize the post-concussion syndrome [31]. Headaches are estimated as occurring variably in 30-90\% of patients who suffered mild head injury [32]. Although, not conclusive, most clinical studies report that PTH occurs less frequently after severe TBI than mild head injury [32, 33].

According to the ICHD-II, headache may develop soon after the trauma and have clinical features similar to primary headaches [34], mostly tension-type headaches, or it may occur late after the injury [35]. However, characteristics of head pain are not required for making diagnosis, in fact this is only made upon the presence of pain temporally related to head injury and adequate instrumental data. The risk of PTH occurrence is higher in women, and increasing age may hamper recovery. Litigation and opportunities of receiving compensation after injuries may support a chronic headache disturbance until the conflict is cleared, although symptoms may not resolve with the settlement of litigation [36]. In our previous study on PTH patients with mild TBI, we found clinical and psychopathological features very similar to those found in primary headaches and no relationship between frequency or severity of headache and pending litigation or resolution of medico-legal controversies [34].

One of the major problems concerning PTH, is that even after mild TBI, the brain is not like it was used to be, no matter whether the injury is detectable or not with sophisticated diagnostic procedure [37]. Therefore, changes in bio-behavioural components must be assumed and neuropsychological and psychopathological clues must be sought as well. The ICHD-II neither provide any neuroradiological, neuropsychological criterion in PTH following mild TBI, nor in the acute or the chronic form. However, the ICHD-II comments to PTH following mild TBI are the following: "Mild head injury may give rise to a symptom complex of cognitive, behavioural and consciousness abnormalities and a GCS of $\geq 13$. It can occur with or without abnormalities in the neurological examination, neuroimaging (CT scan, MRI), EEG, evoked potentials, CSF examination, vestibular function tests and neuropsychological testing. There is no evidence that an abnormality in any of these changes the prognosis or contributes to treatment. These studies should not be considered routine for patients with ongoing post-traumatic headache. They may be considered on a case-by-case basis, or for research purposes". This comment does not apply longer because of the most recent advances in neuroimaging techniques, such as fast sequences MRI, diffusionweighted and diffusion tensor imaging techniques, which may show, at the distance from mild TBI, structural changes in discrete brain areas like frontal and temporal lobes, thalamus, and corpus callosum [37]. Functional techniques approaching cognitive tasks may further clarify metabolic and ischemic damage (secondary injury) after mild TBI [38]. The evaluation of diffuse axonal injury [39] in these patients is promising and may add further data for prognosis after mild TBI [37, 40].

Acute PTH following mild head injury often resolves within few weeks, therefore the major category of PTH patients is represented by those who complain of chronic PTH that may add this symptom to the burden of TBI sequelae for years. Because of the poor knowledge of the pathophysiological mechanisms underlying chronic PTH, an appropriate management is often unavailable. In fact, treatment of chronic PTH is generally difficult and nonspecific and guidelines often resemble those available for primary headaches [41].

It is common belief that mild TBI is often followed by a post-traumatic syndrome including headache and that this syndrome is mostly in accordance with expectation of being entitled with damages. Most of patients who sustained mild TBI seek for medical advice because of their headaches, along with vertigo, dizziness, memory disturbances, sleeping disorders. In most cases this syndrome is the reason why those patients also go through several (and fairly normal) standard diagnostic procedures, and therefore the final diagnosis is likely to be referred to a compensation reward expectation. Such seeking behaviour is therefore considered an indemnity syndrome. However, anxiety and depression due to the traumatic event usually happening in a sudden and unexpected and painful way are the biochemical background for seeking medical reassurance [42]. The development of anxiety and depression may well be a consequence of the trauma rather than a "building up" phenomenon due to expectation. Besides anxiety or depression as symptoms of a psycho-organic syndrome should be taken into account also in mild TBI, given that damage in discrete brain area can be revealed with the appropriate techniques [40]. It is conceivable that this issue calls for medico-legal consideration and for long followup. PTH patients after mild TBI should be thoroughly investigated for psychic disturbances and treated accordingly. Also, difficulties in coping with "a damaged head" should be taken as a symptom to be followed up and cared. An adequate pre-morbid assessment should also be obtained from family and community members. This may also be useful to disclose any personality trait leading to neurotic disorders such as compulsive attitude toward abuse [3]. In fact, a chronic PTH, along with the other symptoms belonging to the post-concussion syndrome, may be sustained by misuse/overuse/abuse of analgesic compounds, either non-opioids, opioids or combination of substances, the latter two being largely responsible for medication overuse headache (ICHD-II code 8.2). To quote 
Sheftell et al. [43]: "It is wrong to oversimplify these complex issues by throwing this disorder into the psychiatric waste basket, simply because we have not adequately described its causes ...".

\section{A glimpse to the future}

Management of PTH is usually based upon the clinical characteristics of the pain which, in most of cases meet the diagnostic criteria for primary headaches, namely migraine and tension-type headache. Accordingly, therapeutic approaches are the same as in the latter conditions. However, results are not satisfactory in the presence of more complex pain syndromes involving psychological as well as organic disorders in head injured patients. PTH classification should therefore be revised in term of pathophysiological mechanisms and studied through neurophysiological and functional neuroimaging techniques along with neuropsychological assessment, rather than in term of temporal pattern. Furthermore, the occurrence of post-traumatic epilepsy [15] in TBI patients complaining of headache and pain, needs to be investigated in order to find common mechanisms that may drive to therapeutic approaches with neuromodulators of neuronal excitability that may show efficacy in post-traumatic syndromes in which pain and epilepsy may be associated.

A systematic review on the prevalence of chronic pain after TBI reported a greater prevalence in civilian patients with mild TBI $(75.3 \%)$ compared with moderate or severe TBI (32.1\%). PTSD may mediate chronic pain, but brain injury appears to have an independent correlation with chronic pain [44].

Susceptibility to primary headache, i.e., personal pretraumatic or family history of head pain should be recollected in TBI patients to better study the evolution and reappearance after TBI and patient pre-TBI personality traits should be obtained to better cope with post-traumatic habit and help with adequate pharmacological and nonpharmacological support.

The combination of psychological changes (depression and anxiety) and organic features (skull fractures, dural lacerations, epileptic EEG abnormalities) in PTH may be inversely correlated with the severity of TBI, with prevalence of psychological disturbances in mild TBI and of organic lesions in severe TBI. Only in severe TBI patients with good cognitive recovery the influence of the psychopathological disorders may play a role. In fact, the affective pain perception is probably related to the integrity of cognitive functions as in mild TBI and in severe TBI with good cognitive outcome.

Due to the great difference between mild, moderate, severe and very severe TBI, attention should be paid to use specific criteria related to coma duration and PTA duration. Epidemiological, clinical and pathophysiological studies should address these issues in order to better categorize and identify PTH in different TBI population.

Conflict of interest None.

\section{References}

1. Headache Classification Committee of the International Headache Society: The International Classification of Headache Disorders 2nd Edition (2004) Cephalalgia 24(Suppl 1):9-160

2. Headache Classification Committee, Olesen J, Bousser MG, Diener HC, Dodick D, First M, Goadsby PJ, Göbel H, Lainez MJ, Lance JW, Lipton RB, Nappi G, Sakai F, Schoenen J, Silberstein SD, Steiner TJ (2006) New appendix criteria open for a broader concept of chronic migraine. Cephalalgia 26:742-746

3. Ferrari A, Coccia C, Sternieri E (2008) Past, present, and future prospects of medication-overuse headache classification. Headache 48:1096-1102

4. Buzzi MG, Cologno D, Formisano R, Rossi P (2005) Prodromes and early phase of migraine attack: therapeutical relevance. Funct Neurol 20:179-183

5. Rossi P, Ambrosini A, Buzzi MG (2005) Prodromes and predictors of migraine attack. Funct Neurol 20:185-191

6. Zasler ND (1999) Posttraumatic headache: caveats and controversies. J Head Trauma Rehabil 14:1-8

7. Baandrup L, Jensen R (2005) Chronic post-traumatic headachea clinical analysis in relation to the International Headache Classification 2nd Edition. Cephalalgia 25:132-138

8. Headache Clssification Committee of the International Headache Society (1988) Classification and diagnostic criteria for headache disorders, cranial neuralgias and facial pain. Cephalalgia 8(Suppl 7): $1-96$

9. Russel WR, Smith A (1961) Post traumatic amnesia in closed head injury. Arch Neurol 5:4-17

10. Jennet B, Frankowski F (1990) The epidemiology of brain injury. In: Handbook of clinical neurology, vol 57. pp 1-16

11. Teasdale G, Jennett B (1974) Assessment of coma and impaired consciousness. A practical scale. Lancet 13:81-84

12. Formisano R, Carlesimo GA, Sabbadini M, Loasses A, Penta F, Vinicola V, Caltagirone C (2004) Clinical predictors and neuropsychological outcome in severe traumatic brain injury patients. Acta Neurochir 146:457-462

13. Formisano R, Bivona U, Penta F, Giustini M, Buzzi MG, Ciurli P, Matteis M, Barba C, Della Vedova C, Vinicola V, Taggi F (2005) Early clinical predictive factors during coma recovery. Acta Neurochir Suppl. 93:201-205

14. Ahmed S, Bierley R, Sheikh JI, Date ES (2000) Post-traumatic amnesia after closed head injury: a review of the literature and some suggestions for further research. Brain Inj 14:765-780

15. Formisano R, Barba C, Buzzi MG, Newcomb-Fernandez J, Menniti-Ippolito F, Zafonte R, Vinicola V, Spanedda F (2007) The impact of prophylactic treatment on post-traumatic epilepsy after severe traumatic brain injury. Brain Inj 21:499-504

16. Fleminger S, Greenwood RJ, Oliver DL (2006) Pharmacological management for agitation and aggression in people with acquired brain injury. Cochrane Database Syst Rev 18:CD003299

17. Ippolito E, Formisano R, Caterini R, Farsetti P, Penta F (1999) Operative treatment of heterotopic hip ossification in patients with coma after brain injury. Clin Orthop Relat Res 365:130 138 
18. Buzzi MG, Tassorelli C, Nappi G (2003) Peripheral and central activation of trigeminal pain pathways in migraine: data from experimental animal models. Cephalalgia 23(Suppl 1):1-4

19. Nozaki K, Boccalini P, Moskowitz MA (1992) Expression of c-fos-like immunoreactivity in brainstem after meningeal irritation by blood in the subarachnoid space. Neuroscience 49:669-680

20. Burstein R (2001) Deconstructing migraine headache into peripheral and central sensitisation. Pain 89:107-110

21. Leone M, Franzini A, Broggi G, May A, Bussone G (2005) Therapeutic stimulation of the hypothalamus: pathophysiological insights and prerequisites for management. Brain 128(Pt 8):E35

22. Missori P, Miscusi M, Formisano R, Peschillo S, Polli FM, Melone A, Martini S, Paolini S, Delfini R (2006) Magnetic resonance imaging flow void changes after cerebrospinal fluid shunt in post- traumatic hydrocephalus: clinical correlations and outcome. Neurosurg Rev 29:224-228

23. Walker WC, Seel RT, Curtiss G, Warden DL (2005) Headache after moderate and severe traumatic brain injury: a longitudinal analysis. Arch Phys Med Rehabil 86:1793-1800

24. Buzzi MG, Bivona U, Matteis M, Spanedda F, Formisano R (2003) Cognitive and psychological patterns in post-traumatic headache following severe traumatic brain injury. Cephalalgia 23:672 (P4L22)

25. Formisano R, Buzzi MG, Bivona U, Matteis M, Caltagirone C (2002) Assessing cognitive and psychological patterns in posttraumatic headache following severe brain injury. J Neurotrauma 19:1366

26. Yehuda R, LeDoux J (2007) Response variation following trauma: a translational neuroscience approach to understanding PTSD. Neuron 56:19-32

27. Kessler RC, Sonnega A, Bromet E, Hughes M, Nelson CB (1995) Posttraumatic stress disorder in the National Comorbidity Survey. Arch Gen Psychiatry 52:1048-1060

28. King NS (2008) PTSD and traumatic brain injury: folklore and fact? Brain Inj 22:1-5

29. Bryant RA, Marosszeky JE, Crooks J, Baguley I, Gurka J (2000) Coping style and post-traumatic stress disorder following severe traumatic brain injury. Brain Inj 14:175-180

30. Bryant RA, Marosszeky JE, Crooks J, Gurka JA (2004) Elevated resting heart rate as a predictor of posttraumatic stress disorder after severe traumatic brain injury. Psychosom Med 66:760-761

31. Evans RW (1996) The postconcussion syndrome and the sequelae of mild head injury. In: Evans RW (ed) Neurology and trauma. W.B. Saunders, Philadelphia, pp 91-116
32. Bazarian JJ, Wong T, Harris M (1999) Epidemiology and predictors of post-concussive syndrome after minor head injury in an emergency population. Brain Inj 13:173-189

33. Minderhoud JM, Boelens MEM, Huizenga J, Saan RJ (1980) Treatment of minor head injuries. Clin Neurol Neurosurg $82: 127-140$

34. Fioravanti M, Ramelli L, Napoleoni A, Lazzari R, Ruggeri S, Cerbo R, Formisano R, Grillo G, Feliciani M, Agnoli A, Spissu A, De Luca P, Merli S (1983) Post-traumatic headache: neuropsychological and clinical aspects. Cephalalgia 3(Suppl 1):221224

35. Evans RW (2004) Post-traumatic headache. Neurol Clin N Am 22:237-249

36. Packard RC (1992) Posttraumatic headache: permanence and relationship to legal settlement. Headache 32:496-500

37. McAllister TW, Saykin AJ, Flashman LA, Sparling MB, Johnson SC, Guerin SJ, Mamourian AC, Weaver JB, Yanofsky N (1999) Brain activation during working memory 1 month after mild traumatic brain injury: a functional MRI study. Neurology 12:1300-1308

38. Metting Z, Rodiger LA, De Keyser J, van der Naalt J (2007) Structural and functional neuroimaging in mild-to-moderate head injury. Lancet Neurol 6:699-710

39. Adams JH, Doyle D, Ford I, Gennarelli TA, Graham DI, McLellan DR (1989) Diffuse axonal injury in head injury: definition, diagnosis and grading. Histopathol 15:49-59

40. Bazarian JJ, Zhong J, Blyth B, Zhu T, Kavcic V, Peterson D (2007) Diffusion tensor imaging detects clinically important axonal damage after mild traumatic brain injury: a pilot study. J Neurotrauma 24:1447-1459

41. Lane JC, Arciniegas DB (2002) Post-traumatic headache. Curr Treat Options Neurol 4:89-104

42. Martelli MF, Grayson RL, Zasler ND (1999) Posttraumatic headache: neuropsychological and psychological effects and treatment implications. J Head Trauma Rehabil 14:49-69

43. Sheftell FD, Tepper SJ, Lay CL, Bigal ME (2007) Post-traumatic headache: emphasis on chronic types following mild closed head injury. Neurol Sci 28(Suppl 2):203-207

44. Nampiaparampil DE (2008) Prevalence of chronic pain after traumatic brain injury: a systematic review. JAMA 300:711-719 